\section{PERMEABILIDADE DO SOLO SATURADO NAS ENCOSTAS DA SUB-BACIA DO RIO MARACANÃ.}

\author{
Francisco Afonso Cavalcanti Júnior ${ }^{1}$
}

Ediléa Dutra Pareira ${ }^{2}$

\begin{abstract}
RESUMO
O presente trabalho tem por objetivo estudar a dinâmica da água na Sub-bacia Hidrográfica do Rio Maracanã, para isso foram realizados ensaios de infiltração e levantamento das características físicas do solo, a fim de correlaciona-los em uma análise dos impactos ambientais decorrentes da expansão urbana na referida bacia. Neste estudo de caso foi adotada como análise a abordagem do método hipotético-dedutivo, o qual visa a descrição do objeto em estudo através da formulação de hipóteses e deduções, conforme Sposito (2004). Foi adotada a metodologia da técnica do Infiltrômetro de duplo anel, conforme Reichardt (1990) e Martins (1976), que consiste na utilização de dois cilindros concêntricos de 60 e $30 \mathrm{~cm}$ de diâmetro, nos quais a água é adicionada, com alturas diferenciadas. Os resultados mostraram o reflexo da produção do espaço urbano dentro da bacia. Os pontos I-1 e I2 estão localizados a nordeste e leste, justamente onde o processo de ocupação da terra é mais intenso, tanto por residências quanto por empreendimentos do Distrito Industrial de São Luís, ao passo que no centro e na direção noroeste da bacia, pontos I-7 e I-10, este processo é menor. As consequências do escoamento superficial em solos expostos e compactados, além dos danos às casas situadas nas direções mais baixas, podem estar relacionadas ao transporte de sedimentos para dentro dos cursos hídricos que vão assim, gradativamente, os assoreando.
\end{abstract}

PALAVRAS-CHAVE: Infiltração de água. Solo. Permeabilidade.

\section{PERMEABILITY SOIL SATURATED ON SLOPES OF SUB- BASIN OF RIO MARACANÃ.}

\begin{abstract}
This paper aims to study the dynamics of water in the Maracanã River sub-basin, for this it was performed infiltration tests and raised the physical characteristics of the soil in order to correlate them in an analysis of the environmental impacts of urban sprawl in basin. In this specific study was adopted the approach of the hypothetical-deductive method, which seek the object description by formulating hypotheses and deductions, as Sposito (2004). It was adopted the methodology of the technique of double ring infiltrometer according to Reichardt (1990) and Martin (1976), in which is use two concentric cylinders with 60 and $30 \mathrm{~cm}$ in diameter, where water is added until differentiated heights. The results showed the reflection of the urban space production within the basin. The points I1 and I-2 are located in the northeast and east, exactly where land occupation process is more intense, both as residences and for projects in the St. Louis Industrial District, while in the center and the northwest of the watershed, points I-7 and I-10, this process is smaller. The consequences of
\end{abstract}

\footnotetext{
${ }_{1}^{1}$ Mestrando, Universidade Estadual Paulista - UNESP/FCT. afonso.jr1@hotmail.com.

2 Docente, Universidade Federal do Maranhão - UFMA. edileap@gmail.com.
} 


\section{Periódica Eletrânica

surface runoff in bare and compacted soils, in addition to damage to the houses located in the lower direction, may be related to sediment transport into the water courses and so gradually the silting

KEYWORDS: Water infiltration. Soil. Permeability.

\section{SUELO PERMEABILIDAD SATURADO EN CUESTAS DE SUBCUENCA DE RIO MARACANÁ.}

\section{RESUMÉN}

Este trabajo tiene como meta estudiar la dinámica del agua en la Subcuenca Hidrográfica del Río Maracanã, para eso fueran realizadas pruebas de infiltración y el lavantamineto de las características físicas del suelo con el fin de correlacionar en un análisis de los impactos ambientales de la expansión urbana en la cuenca. En este estúdio específico fue adoptado como un enfoque analítico el método hipotético-deductivo, que tiene como objetivo la descripción del objeto en estudio por médio de formulación de hipótesis y deducciones, conforme Sposito (2004). Para aquisición de los datos fue adoptado la metodología de la técnica de infiltrómetro de doble anillo conforme Reichardt (1990) y Martin (1976), que es el uso de dos cilindros concêntricos de 60 y $30 \mathrm{~cm}$ de diámetro, donde se añade agua a ambos, com alturas diferenciadas. Los resultados mostraron el reflejo de la producción del espacio urbano dentro de la cuenca. Los puntos de E-1 y E-2 se encuentran al noreste y al este, precisamente donde el proceso de ocupación de tierra es más intensa, tanto como residencias para proyectos en el Distrito Industrial de São Luís, mientras que en el centro y el noroeste de la cuenca, secciones I-7 y la I-10, este proceso es bajo. Las consecuencias de la escorrentía superficial en suelos expuestos y compactados, además de daños a las viviendas ubicadas en la dirección más baja, pueden estar relacionados con el transporte de sedimentos en los cursos de agua y así gradualmente sedimentandolos.

PALABRAS-CLAVE: Infiltración de agua. Suelo. Permeabilidad.

\section{INTRODUÇÃO}

O crescimento populacional, bem como o desenvolvimento econômico, tem implicado em severas alterações no ambiente em decorrência dos usos que são feitos. Tais usos incorrem, quase, sempre em efeitos que se refletem nos danos à qualidade das águas superficiais e subterrâneas.

Áreas que deveriam ser conservadas para a manutenção do sistema hidrológico passam a ser especuladas e ocupadas causando déficit de água em várias localidades e/ou poluição e contaminação das mesmas, em decorrência do mau uso e ocupação da terra gerando-se, dessa forma, prejuízos aos recursos hídricos.

A Sub-bacia do Rio Maracanã se insere neste contexto, apresentando significativa importância para o abastecimento d'água na llha do Maranhão. 
Localizada na cidade de São Luís/MA/Brasil, esta pertence à Bacia Hidrográfica do Rio Bacanga e contribui para o abastecimento da comunidade do Maracanã e das comunidades do entorno. Como características dominantes, a área em estudo apresenta tabuleiros de recarga de aquífero e mananciais circundados por matas ciliares, com buritizais e juçarais que auxiliam na subsistência das populações.

Com uma área de $25 \mathrm{~km}^{2}$, a Sub-bacia do Rio Maracanã é uma das que integram a Bacia Hidrográfica do Rio Bacanga, no município de São Luís, Estado do Maranhão. Está situada entre as coordenadas Universal Transverse of Mercator (Projeção UTM), fuso 23S; N 9706000/ 9713000 m e E 577000/ 584000 m. (Figura 1)

Figura 1: Localização Sub-bacia do Rio Maracanã, São Luís/Maranhão/Brasil.

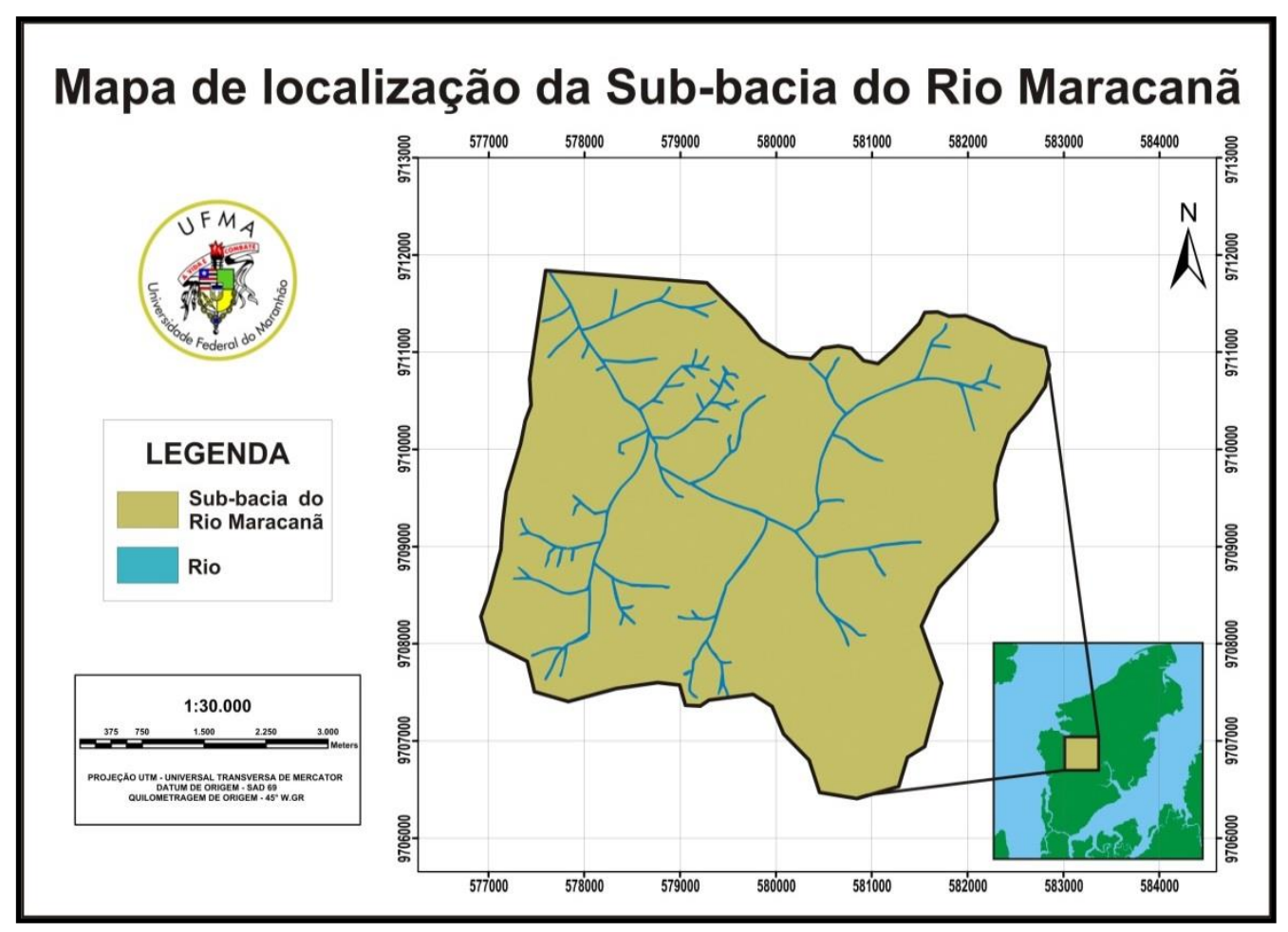

Fonte: Dados da pesquisa.

O acesso a área dar-se pelas bifurcações da BR-135, lado leste, através das vias de acesso ás comunidades do entorno do Distrito Industrial de São Luís, e lado oeste, através da via de acesso da Vila Maranhão.

O presente trabalho tem por objetivo estudar a dinâmica da água na Subbacia Hidrográfica do Rio Maracanã, através de ensaios de infiltração e levantamento das características físicas do solo, a fim de correlaciona-los em uma análise dos impactos ambientais decorrentes da expansão urbana na referida bacia. 


\section{METODOLOGIA}

A base científica para o seu desenvolvimento foi a abordagem teóricometodológica do método hipotético-dedutivo em Geografia, que visa a descrição do objeto em estudo através da formulação de hipóteses e deduções, conforme Sposito (2004).

Foi adotada a metodologia da técnica do infiltrômetro de duplo anel, conforme Reichardt (1990) e Martins (1976), que consiste na utilização de dois cilindros concêntricos de 60 e $30 \mathrm{~cm}$ de diâmetro, onde a água é adicionada em ambos, com alturas diferenciadas, sendo que a maior altura de água será sempre no infiltrômetro interno, para garantir a geração de maior pressão hidrostática. A função do cilindro externo é de atenuar o efeito da histerese no solo e garantir um fluxo vertical no cilindro interno. (Figura 2)

Figura 2. Esquema de infiltração com infiltrômetro de duplo anel.

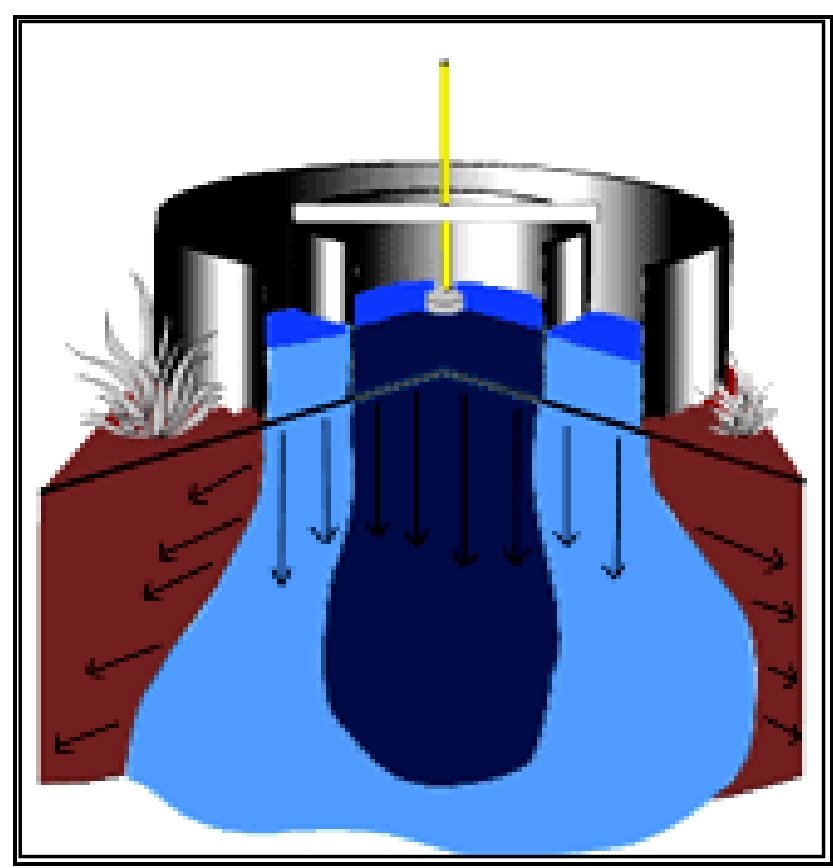

Fonte: http://www.aquagri.com/equipamentos/image equipamentos/doublering4.gif 
A permeabilidade do solo saturado (K), ou condutividade hidráulica, é o coeficiente que expressa a facilidade com que um fluido é transportado através de um meio poroso e depende das propriedades do meio e do fluido (LIBARDI, 1980; CEDERGREN, 1989). É um dos mais importantes parâmetros hidráulicos e depende da natureza do meio, densidade e viscosidade do fluido, sob determinadas condições de temperatura e pressão, em condições de solo saturado o valor de $\mathrm{K}$ é maximo.

A equação utilizada para calcular o valor da permeabilidade do solo saturado (K), foi a proposta por Radcliffe e Simunek (2010) associada aos critérios de classificação de K de Reichardt (1990). Portanto:

$$
K=\frac{I s}{1+\frac{4 \cdot \lambda c}{\pi \cdot r}}
$$

Onde:

K: permeabilidade do solo saturado;

Is: infiltração estável;

$\lambda c$ : comprimento da capilaridade macroscópica do solo conforme as categorias de textura e estrutura;

$\pi: 3,14 ; \boldsymbol{r}$ : raio do anel.

Pode-se determinar a velocidade de infiltração (Vi) para cada instante, considerando os tempos $(\Delta t)$ e os valores de altura $(\Delta h)$, valores estes que são medidos por meio de uma régua fixa no cilindro interno, que indica altura de água sobre o solo. A velocidade de infiltração $(\mathrm{Vi})$ é expressa pela seguinte equação:

$$
V i=\frac{\Delta h}{\Delta t} \times 60=\frac{h_{n}-h_{n-1}}{t_{n}-t_{n-1}} \times 60
$$


O gráfico resultante da infiltração acumulada $(\Delta \mathrm{h})$ versus tempo $(\Delta \mathrm{t})$ indica as medidas de $\mathrm{Vi}$, grande no início da infiltração e que vai diminuindo com o tempo. $\mathrm{O}$ solo se satura e a velocidade de infiltração se estabiliza, assumindo um valor constante, denominado permeabilidade do solo saturado (K). (Tabela 1)

\begin{tabular}{l|c} 
Tabela 1: Critério de classificação de infiltração básica. \\
\hline $\mathbf{K}$ & VALOR $\mathbf{( c m} / \mathbf{h})$ \\
\hline Muito alto & 3 \\
Alto & $1,5-3$ \\
Médio & $0,5-1,5$ \\
Baixo & $0,1-0,5$ \\
Muito baixo & $<0,1$ \\
\hline
\end{tabular}

Fonte: Reichardt ,1990.

O movimento da água no meio poroso torna-se muito complexo devido à alta variabilidade das propriedades física dos solos e rochas. A permeabilidade apresenta uma distribuição de frequência log-normal devido a processos químicos, físicos e biológicos que ocorrem continuamente no solo como, por exemplo, a alteração de íons trocáveis, a migração de partículas de argila durante o fluxo, presença de macroporos, pedoperturbação e outros.

A análise granulométrica das amostras de solo foi realiza pelo Laboratório de Mecânica dos Solos do Instituto Federal de Educação, Ciência e Tecnologia do Maranhão - IFMA.

\section{RESULTADOS E DISCUSSÕES}

As encostas dos tabuleiros dissecados apresentaram no horizonte " $A$ " coloração variável de 5Y 7/2 (cinza-claro) a 2,5YR 6/4 (bruno - avermelhado claro) com granulometria na fração areia $(81,4$ a $98,6 \%)$, silte $(1,4$ a $11,8 \%)$ e argila $(0,02$ a 6,77\%), indicando o solo arenoso, conforme classificação IBGE (2007). Registrase a ocorrência de uma camada espessa de concreções lateríticas e de plintita de forma difusas e/ou concentrada no horizonte "B" do solo. 
O solo pode atingir uma espessura menor que $1 \mathrm{~m}$. O limite de liquidez varia de $12,41 \%$ a $15,18 \%$ e de plasticidade de $16,12 \%$ a $18,31 \%$, indicando um índice de consistência (IC) dura e índice de plasticidade não plástica. (Tabela 2)

Tabela 2: Análise granulométrica encosta de tabuleiro.

\begin{tabular}{l|l|l|l|l|l|l|l|l|l|l}
\hline \multirow{2}{*}{ Amostra } & \multicolumn{5}{|c|}{$\%$ em peso } & \multicolumn{3}{|c}{ Propriedade do solo } \\
\cline { 2 - 12 } & Peso (g) & Areia & Silte & Argila & LL(\%) & LP(\%) & IP(\%) & IC & C & P \\
\hline $\mathrm{I}-2$ & 200 & 81,41 & 11,83 & 6,77 & 12,41 & 16,12 & $-3,71$ & 1,35 & Dura & NP* \\
\hline $\mathrm{I}-7$ & 201 & 95,22 & 4,76 & 0,02 & 15,18 & 18,31 & $-3,13$ & 1,88 & Dura & $N P^{*}$ \\
\hline $\mathrm{I}-10$ & 201 & 98,58 & 1,42 & 0,00 & 15,88 & 19,66 & $-3,78$ & - & - & - \\
\hline
\end{tabular}

Fonte: Dados da pesquisa.

A altitude das encostas varia de 25 a 39 metros, com declividade oscilando entre moderadamente a fortemente ondulado. As encostas encontram-se intensamente antropizados, em geral ocupadas por residências, sendo que na porção oeste da sub-bacia há presença de extração de lateritas, o que causa o surgimento de ravinas e voçorocas na área e o assoreamento dos rios e igarapés da região. A vegetação predominante é a do tipo secundária (capoeira média e alta), além de árvores frutíferas como o cajueiro e a mangueira.

Os dados obtidos nos ensaios de infiltração forneceram informações acerca da dinâmica do comportamento e capacidade de infiltração do solo nesta unidade da paisagem através do infiltrometro de duplo anel.

Os ensaios de infiltração (I-1, I-2, I-7, e I-10) nas encostas do tabuleiro dissecado, apresentaram valores de volume acumulado variando de $2755,35 \mathrm{~cm}^{3}$ a $26211,15 \mathrm{~cm}^{3}$ (cerca de 3,0 a 26,21 litros) em, aproximadamente, 1,5 hora. (Figuras 4) 


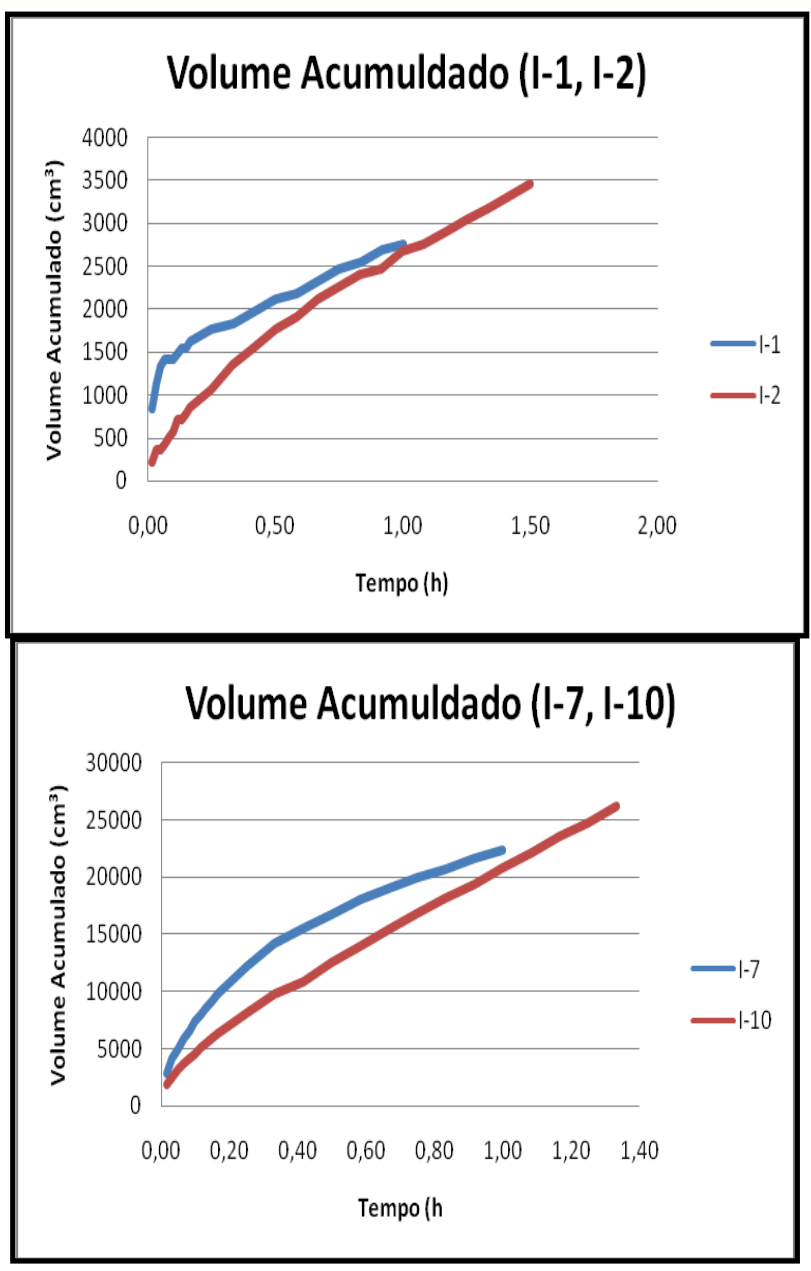

Fonte: Dados da pesquisa.

A permeabilidade do solo saturado $(K)$ apresentou resultados anômalos, nos quais os pontos $\mathrm{I}-1$ e I-2 obtiveram o valor de $\mathrm{K}=0,11 \mathrm{~cm} / \mathrm{h}$, e os pontos $\mathrm{I}-7$ e I-10 obtiveram os valores respectivamente de $K=0,70 \mathrm{~cm} / \mathrm{h}$ e $\mathrm{K}=1,11 \mathrm{~cm} / \mathrm{h}$, observa-se que esses resultados configuram-se como discrepantes para unidades de paisagem na mesma área. Analizando-os, segundo os critérios de classificação de Reichardt (1990), os dois primeiros apresentaram valores de K considerados como baixos, destaca-se que a superfície do solo encontrava-se exposto e compactado e com alto nível de antropização, e os dois últimos tiveram os seus valores de $\mathrm{K}$ considerados como médios, nestes foi registrado a presença de lateritas. Destaca-se que a ocorrencia de lateritas facilita a distribuição de macrosporos no solo (Figura 5). 
Figura 5. Superfície do solo compactada no ponto I-1 (esquerda), solo da encosta com concreções lateriticas no ponto I-7 (direita).

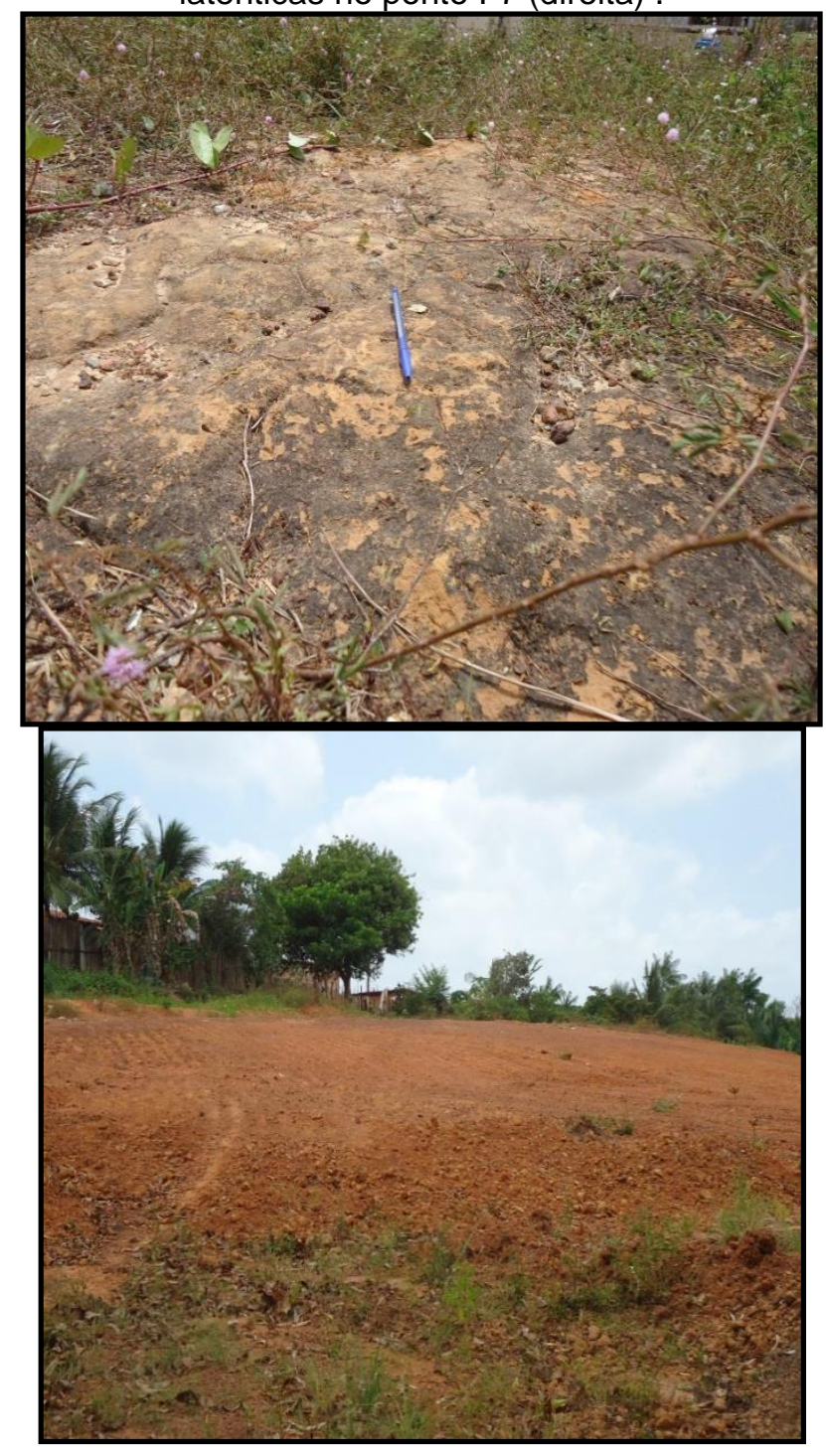

Fonte: Dados da pesquisa.

O grau de impermeabilização da superfície do solo influenciou diretamente na velocidade de infiltração, que no início dos experimentos apresentou taxas distintas de velocidade. Os ensaios nos pontos $\mathrm{I}-1$ e $\mathrm{l}-2$ tiveram as menores velocidades iniciais de infiltração $(72,0 \mathrm{~cm} / \mathrm{h}$ e $18,0 \mathrm{~cm} / \mathrm{h})$ e os ensaios $\mathrm{I}-7$ e $\mathrm{I}-10$ as maiores velocidades iniciais $(240,0 \mathrm{~cm} / \mathrm{h}$ e $162 \mathrm{~cm} / \mathrm{h})$ para a unidade de paisagem considerada (Figura 6). 
Figura 6: Curva de velocida de infiltração em encosta da colina, pontos I-1, I-2 (esquerda) e pontos I-7,I-10 (direita).

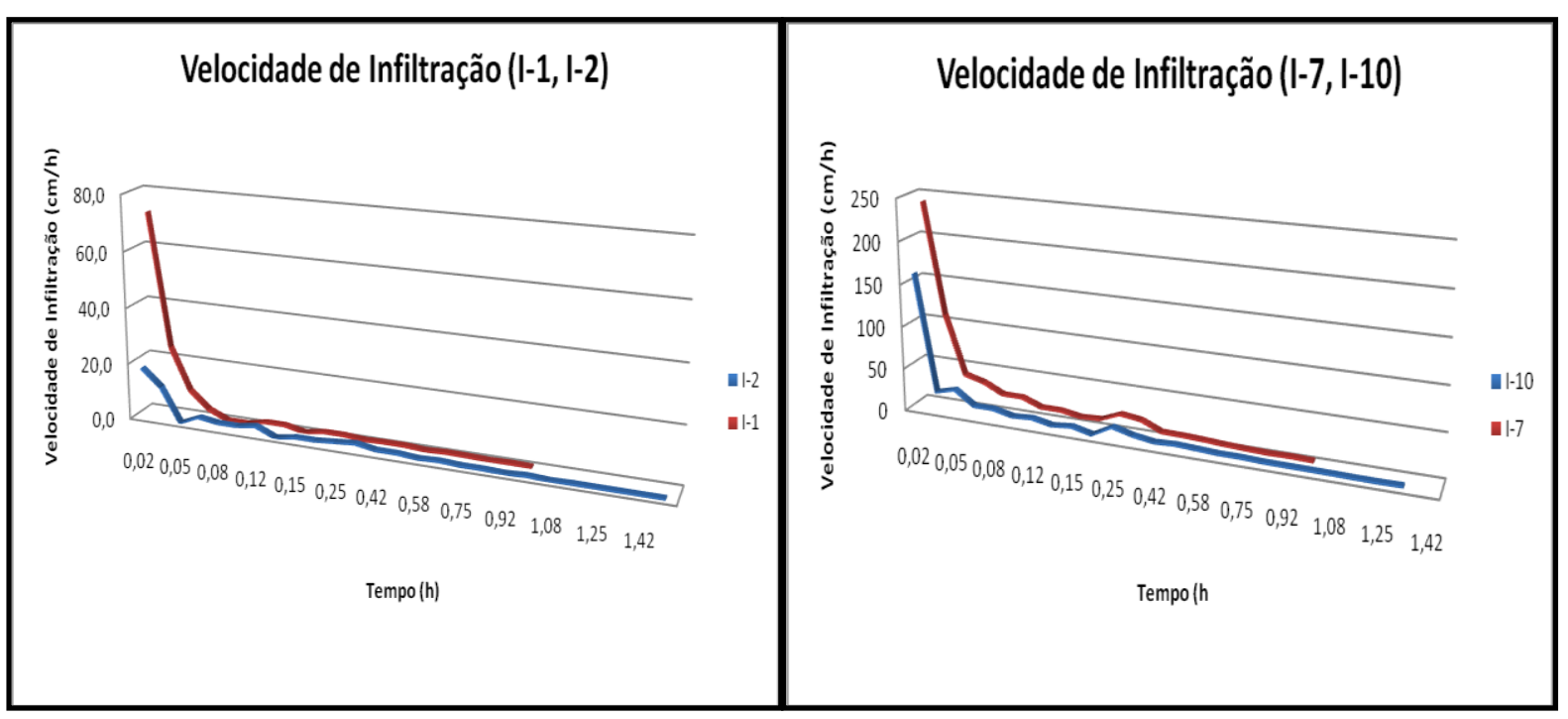

Fonte: Dados da pesquisa.

Contudo, ao atingirem o grau de saturação, para todos os quatro experimentos, a velocidade de infiltração atingiu em aproximadament 1,5 horas um valor de $0,2 \mathrm{~cm} / \mathrm{h}$ a $0,3 \mathrm{~cm} / \mathrm{h}$, exceto para o ensaio $\mathrm{I}-10$ que apresentou $3 \mathrm{~cm} / \mathrm{h}$.

\section{CONCLUSÃO}

Os resultados mostraram o reflexo da produção do espaço urbano dentro da bacia. Os pontos I-1 e I-2 estão localizados a nordeste e leste, justamente onde o processo de ocupação da terra é mais intenso, tanto por residências quanto por empreendimentos do Distrito Industrial de São Luís, ao passo que no centro e na direção noroeste da bacia, pontos I-7 e I-10, este processo é menor. Neste cenário o volume acumulado de infiltração foi considerado respectivamente baixo e médio.

Nos pontos de médio volume acumulado de infiltração pode haver uma maior contribuição para a manutenção do fluxo de base e do lençol freático, devido a presença de materiais lateríticos nos solos dessas áreas. O mesmo não se pode inferir dos pontos de baixo volume acumulado, pois 0 alto grau de impermeabilização dos solos favorece o escoamento superficial antes mesmo de atingir o potencial de saturação. 
Sendo assim, as consequências do escoamento superficial em solos expostos e compactados, além dos danos às casas situadas nas direções mais baixas, podem estar relacionadas ao transporte de sedimentos para dentro dos cursos hídricos e assim, gradativamente os assoreando.

\section{BIBLIOGRAFIA}

CEDERGREN, H. R.. Seepage, drainage and flow nets. John Willey and Sons. New York, 1989.

INSTITUTO BRASILEIRO DE GEOGRAFIA E ESTATístICA - IBGE. Manual técnico de Pedologia. Manuais técnicos em Geociências. Número 4.(2ª edição). Diretoria de Geociências. Rio de Janeiro, 2007.

LIBARDI, P. L.. Dinâmica da água no solo. ESALQ/USP. Piracicaba, 1980.

MARTINS, J. A. Infiltração.(p. 44 - 55). In: PINTO, N. L. de S.; HOLTZ, A. C. T.; MARTINS, J. A.; GOMIDE, F. L. S.. Hidrologia Básica. Edgard Blucher. São Paulo, 1976.

RADCLIFFE, D. E.; SIMUNEK, J.. Soil physics with hydrus: modeling and applications. CRC Press. Florida, 2010.

REICHARDT, K.. A água em sistemas agrícolas. Manole. São Paulo, 1990.

SPOSITO, E. S.. Geografia e Filosofia: contribuição para o ensino do pensamento geográfico. Editora UNESP. São Paulo, 2004. 